\title{
Low-Cost Online Contact Resistance Measurement of Power Connectors to Ease Predictive Maintenance
}

\author{
A. Kadechkar, M. Moreno-Eguilaz, J.-R. Riba, Member, IEEE, and F. Capelli
}

\begin{abstract}
With the increasing use of sensors and wireless communication systems, predictive maintenance is acquiring more and more importance to assess the condition of in-service equipment. Predictive maintenance presents promising cost savings, as it allows minimizing unscheduled power systems faults, which can have very costly and catastrophic consequences. Early stage detection of power system failure requires acquiring, monitoring, and periodically analyzing the condition of the elements involved, such as high-voltage power connectors, since they are critical devices which are often located in key points of power systems. This paper proposes a low-cost online system to determine the contact resistance of high-voltage direct current (DC) and alternating current (AC) power connectors, to determine their health condition in order to apply a predictive maintenance plan. The contact resistance is considered as a reliable indicator of connector's health condition. However, it cannot be directly measured, and the applied strategy differs between DC and AC power systems. Experimental results show a maximum error of $5 \%$, thus proving the accuracy and feasibility of the approach presented in this paper, since the proposed limit of acceptable resistance increase is $20 \%$. This approach can also be applied to many other power systems' elements.
\end{abstract}

Index Terms-Connector, contact resistance, finite element, predictive maintenance, online measurement.

\section{INTRODUCTION}

$\mathrm{P}$ OWER connectors are critical elements in electrical power grids. Failure of such elements can cause severe outages with catastrophic and costly consequences [1]. Utilities and system operators must ensure a safe, reliable and continuous delivery of power to customers, while trying to minimize any outage in the service [2]. Power connectors are electromechanical devices [3] intended to provide stable and

A. Kadechkar is with the Universitat Politècnica de Catalunya, Electrical Engineering Department, Rambla Sant Nebridi 22, 08222 Terrassa, Spain (email: akash.kadechkar@upc.edu)

M. Moreno-Eguilaz is with the Universitat Politècnica de Catalunya, Electrical Engineering Department, Rambla Sant Nebridi 22, 08222 Terrassa, Spain (e-mail: manuel.moreno.eguilaz@upc.edu)

J.-R. Riba is with the Universitat Politècnica de Catalunya, Electrical Engineering Department, Rambla Sant Nebridi 22, 08222 Terrassa, Spain (email: riba@ee.upc.edu)

F. Capelli is with SBI Connectors España, C. Albert Einstein, 5 - 7 Polígono Industrial Sesrovires, 08635 Sant Esteve de Sesrovires, Spain (email: francesca.capelli@sbiconnect.es) reliable connection and to transmit electrical power between different conductors or bus bars [4], while minimizing the voltage drop and the associated power losses. They are usually made of copper or aluminum, the latter material being more commonly used in substation connectors. High-voltage mechanical power connectors are bolted elements, using bolts and nuts, as it is known that this type of connections allow performing reliable and compact contacts [5], although they can produce irregular contact pressure. The number of contacting spots and thus the contact area tends to increase with the mechanical load applied [6]. Apart from the mechanical load, the condition of the contact interface plays a key role on contact resistance [7]. Since the spots provide the conducting pathways for the transfer of electrical current between the two mating surfaces [5], the contact resistance tends to decrease when increasing the nominal contact area [8], and the number of conducting spots within the contact interface [3].

Temperature cycling due to peak and off-peak daily patterns tends to lose the bolts, thus producing an increase of the contact resistance of the connector. It has been reported that re-torqueing is not a useful practice to overcome this issue [9]. When the resistance exceeds a limit value, the connector must be replaced before failure. Improved electrical contacts require reduced and stable contact resistance over time, otherwise overheating and shortening of service life are expected [7]. Predictive maintenance plans allow avoiding major failures of power connectors, although they require diagnosing the condition of such devices. To this end, it is highly appealing to acquire online data, from which it is possible to perform a fast diagnosis of their condition, thus identifying the connectors that will fail prematurely and therefore avoiding severe power system faults [10]. This approach allows replacing or applying a predictive maintenance plan, as soon as the connector shows degraded electrical performance.

The electrical contact resistance determines the efficiency and expected lifetime of power connectors [11], thus being considered a reliable indicator of their health condition. As soon as the contact resistance of the connector increases, both thermal and electrical behaviors, which are interrelated, tend to deteriorate [12]. It is a recognized fact that the contact resistance has two components, the electrical constriction resistance (ECR) and the film resistance (FR). The ECR is produced by the roughness of the mating surfaces, which 
forces the electrical current flowing through a constricted area. This component is affected by the mechanical load applied by the bolting elements, electrical and mechanical properties of the mating surfaces or environmental conditions [13]. The FR is generated by oxides and poorly conductive films formed at the contact interface [14].

Different methods to measure the contact resistance are found in the bibliography. The 4-wire method is probably the most widely accepted method, which has been already used to measure the contact resistance in different connector types [15]-[19]. In [20] an electrothermal model of automotive connectors was proposed and a shunt resistor was used to sense the current. In [21] the $\mathrm{AC}$ contact resistance was measured by using a Rogowski coil and a voltage probe assuming that the inductance of the circuit remained constant. Finally, in [22] it is proposed to analyze the current during a switching process since faults such as loose contacts cause a distortion of the current waveform.

This work proposes a low-cost online approach to measure the electrical contact resistance of high-voltage power connectors focused to ease predictive maintenance plans. In addition, power connectors are often installed outdoors, under high-current and high-voltage environments, where human interventions must be avoided or at least minimized. However, online monitoring on high-voltage systems is still not common [23]. The proposed approach applies a hybrid experimental and numerical approach to determine the contact resistance, which includes finite element method (FEM) simulations. Nowadays, FEM simulations are being commonly used in the area of sensors, especially when analytical equations are difficult to apply [24]-[26]. The proposed approach is valid for both DC and AC applications. The methodology proposed in this work is based on the simultaneous measurement of the current and the voltage drop across the connector. It does not require any current injection from an external source, since the line current passing through the connector is measured, and used to determine the voltage drop. Whereas the current is measured by using a low-cost analog-bipolar Hall effect sensor, the voltage drop is acquired by means of an instrumentation amplifier. At knowledge of the authors, there is a scarcity of works dealing with this application, so this work makes a contribution in this area. Although this work is focused on high-voltage power connectors, the same approach can be applied to a wide range of electrical devices and apparatus, such as cable joints, electrical pantographs, or circuit breakers among others.

\section{ONLINE RESISTANCE MEASUREMENT}

This section analyzes the online approach proposed in this work for determining the contact resistance of power connectors. The online determination of the contact resistance requires the simultaneous measurement of the temperature, the voltage drop and the current across the connector under normal conditions of operation, for both DC and AC power frequency supplies. These measurements are a challenging problem, as the expected current is in the range of $0.1-10 \mathrm{kA}$, whereas the expected voltage drop is of some $\mathrm{mV}$, since the expected contact resistance is in the order of some microohms.

Since the contact resistance of power connectors is in the order of some micro-ohms a suitable and sensitive measurement system is required. The standard 4-wires method is based on injecting a stabilized DC current in the range of 10-200 A. However, this requisite makes it unfeasible to develop a low-cost online measurement system. This paper proposes acquiring and using the own line current to determine the voltage drop across the terminals of the connector, and thus its contact resistance. This approach is also extended to deal with power frequency supply, although, in this case, the measurement of the contact resistance becomes more complex. Under AC supply, when dividing the voltage drop across the connector by the electrical current, the impedance of the connector is obtained instead of the resistance. The impedance can be rather higher than the resistance, because of the reactance component. The values of the reactance and the impedance depend on the geometry of the experimental loop and the frequency of the $\mathrm{AC}$ current.

Online contact resistance determination requires measuring the initial contact resistance of the power connector. During installation, it is usual to measure the DC resistance of the power connection by means of a portable micro-ohmmeter to check that the connector has been correctly installed. Therefore, this work assumes that the initial DC resistance of the connector is known, which will be used as the reference value or starting point.

The initial resistance is initially measured by means of the resistance is commonly done by applying the standard Kelvin or 4-wire method as schematized in Fig. 1, since the results are not influenced by the resistance of the probes [27]. This measurement requires a 4-leads micro-ohmmeter, which injects a stabilized DC current $I_{D C}$ and measures the voltage drop $\Delta V_{D C}$ across the connector.

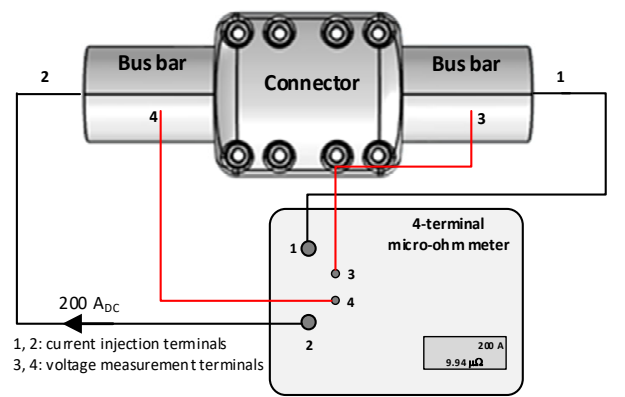

Fig. 1. 4-wires method to measure the total resistance of the connector.

The DC resistance of the connector at the standard temperature of $20{ }^{\circ} \mathrm{C}$ is calculated from the current $I_{D C}$ and the voltage drop $\Delta V_{\text {Connector }}$ as,

$$
R_{\text {Connector }, 20^{\circ} \mathrm{C}}=\left(\Delta V_{\text {Connector }} / I_{D C}\right) /[1+\alpha(T-20)]
$$

where $T\left({ }^{\circ} \mathrm{C}\right)$ is the actual temperature of the connector, and $\alpha_{A l}=0.004 \mathrm{~K}^{-1}$ is the temperature coefficient of aluminum, the material of the connector analyzed in this work.

The contact resistance $R_{\text {Contact }}$ is calculated by subtracting the bulk resistance $R_{B u l k}$ from the total resistance of the power connector $R_{\text {Connector, } 20^{\circ} \mathrm{C}}$ as, 


$$
R_{\text {Contact }}=R_{\text {Connector }, 20^{\circ} \mathrm{C}}-R_{\text {Bulk }}
$$

$R_{\text {Bulk }}$ must be determined offline by means of FEM (finite element method) simulations, since it cannot be measured directly, whereas $R_{\text {Connector }, 20^{\circ} \mathrm{C}}$ must be measured by means of the 4-wires method. The bulk resistance $R_{B u l k}$ depends on the size, shape, and the electrical properties of the connector. It is calculated by means of FEM by injecting a known current and measuring the voltage drop between the terminals of the connector. When injecting a DC current, $R_{B u l k, D C}$ is obtained by means of this approach, whereas when injecting an $\mathrm{AC}$ current the impedance is obtained instead, thus allowing to calculate $R_{B u l k, A C}$ as the real part of the impedance. This strategy enables determining the $R_{A C} / R_{D C}$ ratio, which is required in Section III.B.

Fig. 2 shows a three-dimensional view of the analyzed power connector and bus bar, as well as the mesh applied in the FEM simulations, which includes around 5.9 million of tetrahedral elements, more than 590000 triangular elements and around 33000 edge elements. FEM simulations were performed by means of the Comsol Multiphysics package.

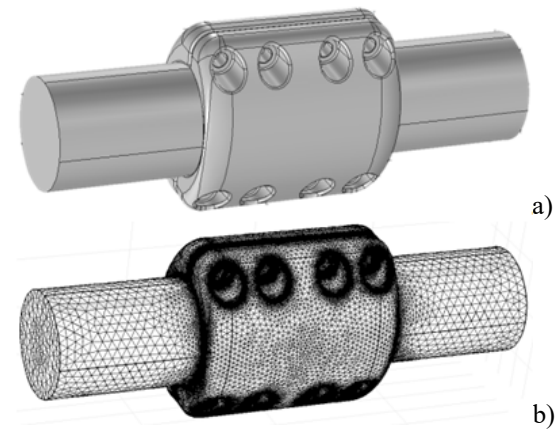

b)

Fig. 2. a) Three-dimensional view of the connector and bus bar. b) Mesh of the connector and bus bar.

It is noted that (1) assumes that the resistance of the connector increases linearly with its temperature and so, it is required a simultaneous measurement of the temperature, voltage drop and current. This work proposes to measure the temperature by using T-type thermocouples installed in the connector and the adjoining bus bar.

The voltage drop across the power connector can be measured by means of an instrumentation amplifier. Since the expected voltage drop is in the order of some $\mathrm{mV}$, it is essential minimizing unwanted noise effects. As there are very small temperature differences between the terminals of the connector, thermo-voltages are not an issue [28]. Apart from rejecting the common-mode noise, the instrumentation amplifier also allows amplifying the input signal. The remaining noise can be further minimized by applying a suitable post-processing filtering.

Different sensors can be applied for an online measurement of the current, including current transformers, Rogowski coils, shunt resistors, Giant magneto impedance (GMI), Giant magneto resistive (GMR), or analog-bipolar Hall effect sensors [29], [30]. However, for the application described in this work, the coreless Hall effect sensor is a suitable choice, since it allows a contactless measurement of both DC and AC magnetic fields, high-current capability, low-cost, reduced size and accuracy [31]. In the technical literature there are few works applying coreless Hall effect sensors in high-current applications. It is usual to deal with sensors including a ferromagnetic core acting as a field concentrator [31], [32], although this option is not practical for the application proposed in this paper. Due to the outer diameter of the bus bars, a bulky, difficult-to-install and expensive magnetic core is required. If the sensitivity factor of the sensor is known, which is usually expressed in $\mathrm{mV} / \mathrm{mT}$, it is straightforward to convert the output voltage of the coreless Hall effect sensor into the current expressed in amps.

According to the Biot-Savart law, the magnetic flux density $B$ generated by a current $I_{\text {Connector }}$ flowing through a rigid bus bar of round cross-section of known diameter $D$, is given by,

$$
B=\frac{\mu_{0} \cdot I_{\text {Connector }}}{\pi D}
$$

and the measured output voltage of the sensor once the offset has been removed can be expressed as,

$$
V_{\text {Hallsensor }}=k B
$$

$k[\mathrm{~V} / \mathrm{T}]$ being the sensitivity constant.

Thus, when placing the sensor on the surface of the bus bar, the current through the conductor and hence, through the connector, can be calculated as,

$$
I_{\text {Connector }}=\frac{B \cdot D}{4 \cdot 10^{-7}}
$$

Finally, from (4) and (5) the current is obtained as,

$$
I_{\text {Connector }}=\frac{V_{\text {Hallsensor }} \cdot D}{k \cdot 4 \cdot 10^{-7}}
$$

Sensitivity changes due to gap, diameter or sensor orientation tolerances are the main error causes of open-loop Hall effect sensors [32]. This error can be minimized by means of an in situ calibration of the sensor once installed.

\section{Proposed Online Methods To Determine The CONTACT RESISTANCE OF THE CONNECTOR}

It is easier to measure the contact resistance under DC than under AC supply. This is because there are some effects that do not arise under DC supply, such as eddy currents or inductive effects due to the parasitic reactance induced around the conductor.

\section{A. Determination of the Contact Resistance under DC Supply}

The contact resistance of aluminum connectors can be greater than the bulk resistance [16]. The principle to measure the contact resistance is based on measuring the total resistance of the connector, and afterwards subtracting the bulk resistance component.

The total resistance can be measured experimentally. However, the bulk resistance must be calculated, for example by means of FEM simulations, as already explained. The 4wire method has two independent voltage and current circuits, this property being exploited in the online measurement techniques developed in this section. Therefore, the total resistance of the connector $R_{\text {Connector }}$ can be determined from the voltage drop across the connector $\Delta V_{\text {Connector, }}$ and the 
current through the line or the connector $I_{\text {Connector }}$ as,

$$
R_{\text {Connector }}=\frac{\Delta V_{\text {Connector }}}{I_{\text {Connector }}}
$$

To this end, a current high enough to produce a sufficient voltage drop across the connector is required, which can offset the temperature dependent voltage between the voltage measuring leads due to the thermoelectric effect. When dealing with power connectors rated from several hundreds of amps to some kilo-amps, the same line current can be used to determine the voltage drop across the connector.

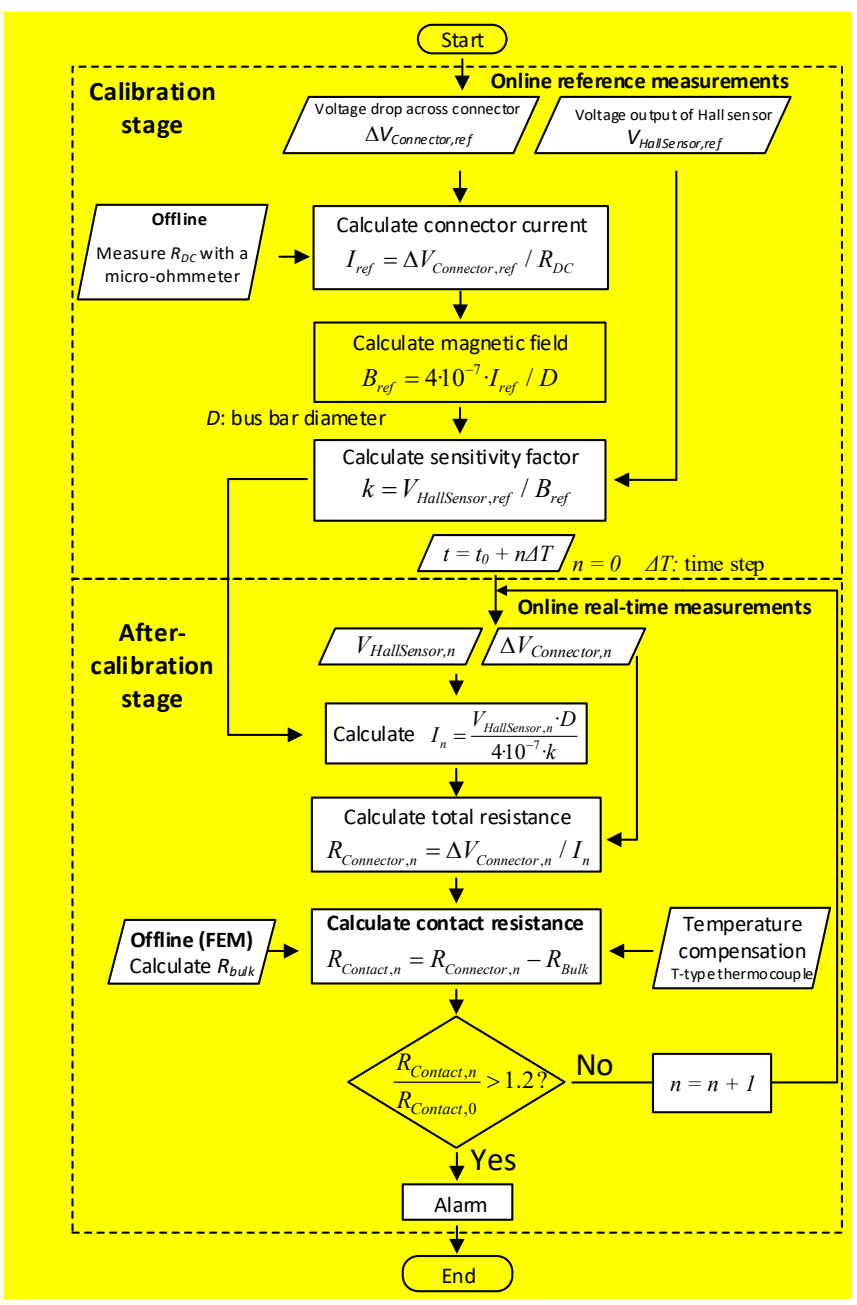

Fig. 3. Flowchart of the online method proposed in this work to determine the electrical contact resistance of the connector under DC supply.

Fig. 3 shows the procedure proposed in this work to determine the contact resistance of the connector under DC supply. This procedure has two main stages, namely calibration and after-calibration stages, respectively. The first stage is carried out during the installation of the power connector. The second one is performed under normal operating conditions, i.e., during the useful life of the power connector. Whereas in the first stage the sensitivity of the Hall effect sensor is calibrated while installing the connector, during the after-calibration stage the actual value of the contact resistance is recalculated and updated based on online measurements.

The DC resistance $R_{D C}$ of the power connector must be measured in situ, before the circuit is connected using a handheld portable micro-ohmmeter. Once the circuit is connected, both the output voltage of the Hall effect sensor and the voltage drop across the connector must be measured to calibrate the sensitivity factor of the sensor. Next, during the after-calibration stage, the instantaneous values of $\Delta V_{\text {Connector }}$ and $V_{\text {HallSensor }}$ are measured to determine the total resistance of the connector, $R_{\text {Connector }}$. Finally, the contact resistance is calculated from (2), once the bulk resistance of the connector $R_{\text {Bulk }}$, is known. It should be noted that $R_{\text {Bulk }}$ must be calculated from FEM simulations only once, during the design and optimization stage of the connector.

When it is no possible an offline measurement of the DC resistance of the connector, it can be estimated from the bulk resistance as $R_{D C} \approx 3 R_{B u l k}$. This assumption is valid for cast A356.0-T6 aluminum connectors when applying the standard installation procedure suggested by the manufacturer [33].

\section{B. Determination of the Contact Resistance under Power Frequency AC Supply}

As already mentioned, under power frequency AC supply, the online measurement of the current and the voltage drop across the connector provides the impedance instead of the AC resistance since, 


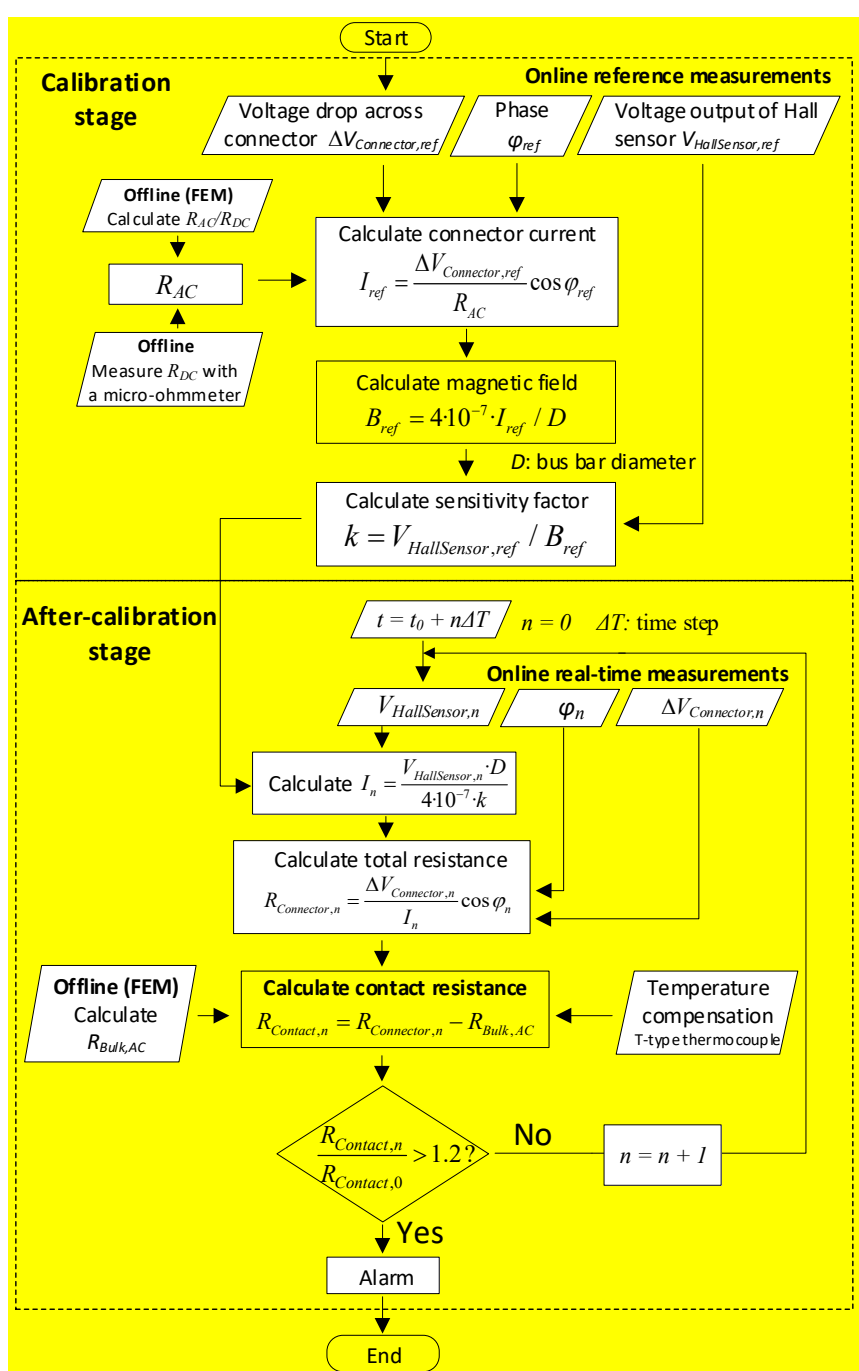

Fig. 4. Flowchart of the online method proposed in this work to determine the electrical contact resistance of the connector under power frequency $\mathrm{AC}$ supply.

$$
Z_{\text {Connector }}=\frac{\Delta V_{\text {Connector }}}{I_{\text {Connector }}}=\frac{k \cdot 4 \cdot 10^{-7} \cdot \Delta V_{\text {Connector }}}{V_{\text {HallSensor }} \cdot D}=\sqrt{R_{\text {Connector }}^{2}+X_{\text {Connector }}^{2}}
$$

In this case, the reactance and thus the $\mathrm{AC}$ resistance, can be estimated from the phase difference between the current and the voltage drop across the connector. It can be done from the online phase difference between $V_{\text {Hallsensor }}$ and $\Delta V_{\text {Connector }}$ [34]-[36]. Fig. 4 summarizes the online procedure to determine the $\mathrm{AC}$ contact resistance of power connectors. As in the DC case, it has two main stages, namely calibration and after-calibration stages. The $\mathrm{AC}$ resistance $R_{A C}$ of the connector is measured offline similarly as done under DC supply, once the $R_{A C} / R_{D C}$ ratio is known from FEM simulations. The voltages $\Delta V_{\text {Connector }}$ and $V_{\text {Hallsensor, and their }}$ phase difference $\varphi$ must be measured to calibrate the sensitivity factor of the Hall effect sensor. Next, during the after-calibration stage, the instantaneous values of $\Delta V_{\text {Connector }}$, $V_{\text {HallSensor }}$ and $\varphi$ are measured to determine the total resistance of the connector, $R_{\text {Connector }}$. Finally, the contact resistance is calculated from (2), once the bulk resistance of the connector, $R_{B u l k, A C}$, is known from FEM simulations. This method is based on,

$$
R_{\text {Connector }}=Z_{\text {Connector }} \cdot \cos \varphi
$$

The impedance of the connector, $Z_{\text {Connector, }}$ is calculated according to (8). It should be noted that (9) can be applied since $V_{\text {HallSensor }}$ is related to the current $I$ by applying (6). Therefore, the waveforms of $V_{\text {HallSensor }}$ and $I$ are in-phase. This assumption was already verified in the laboratory by comparing the output voltage waveform of a calibrated Rogowski coil with that of the Hall effect sensor.

\section{EXPERIMENTAL RESUlTS}

This section develops the experimental part of this work to assess the performance of the proposed approaches for determining the contact resistance of high-voltage power connectors.

A suitable experimental setup is required to measure the resistance of the connector. For online data acquisition, a NI USB-6000 multifunction DAQ from National Instruments was used. The data was monitored and recorded by means of the SignalExpress 2015 software, also from National Instruments.

Two solid cylindrical aluminum bus bars of $1 \mathrm{~m}$ length and $40 \mathrm{~mm}$ diameter each, were electrically joined by means of the analyzed power connector. To determine the contact resistance of the connector, the voltage drop between points 2 and 3 was measured by using the inbuilt instrumentation amplifier of the USB-6000 data acquisition system, as shown in Fig. 5a.

An analog-bipolar DRV5033VA Hall effect sensor from Texas Instruments was used to measure the current through the test loop. It has a sensitivity in the range of 45-140 $\mathrm{mV} / \mathrm{mT}$. The sensor was placed on the top of the bus bar, oriented towards the direction of the magnetic field lines. The output voltage of the Hall effect sensor was acquired by means of the USB-6000 DAQ system.

As described in Section II, the contact resistance must be referred to the standard temperature of $20{ }^{\circ} \mathrm{C}$. Therefore, the temperatures of the bus bars and the connector were acquired simultaneously with the voltage drop and the current, by using T-type thermocouples and an Omega TC-08 thermocouple data logger.

The contact resistance of the connector was determined from online voltage, current and temperature measurements performed by means of the above mentioned devices, and FEM simulations, as detailed in Section II.

Fig. 5a shows the analyzed power connector and the solid aluminum bus bars, whereas Fig. 5b shows the loop used to test the connector.

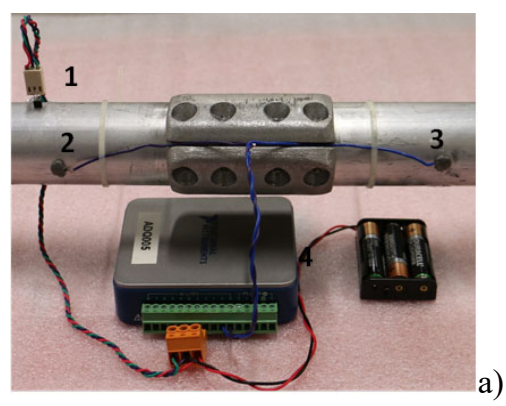




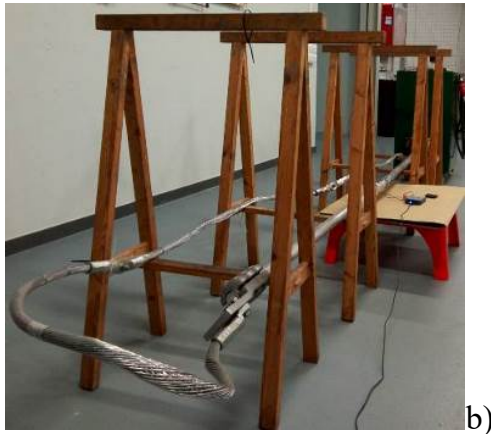

Fig. 5. a) The tested bolted mechanical connector with the entire setup, including bus bars, sensors (1: Hall effect sensor, 2-3: voltage drop terminals, 4: DAQ) to measure and data acquisition modules. b) The loop used to test the power connector.

To test the loop, a stabilized and adjustable DC current source of $0-200 \mathrm{~A}_{\mathrm{DC}}$ was used to ensure a stabilized DC supply, as well a high-current transformer with an output current $0-10 \mathrm{kA}_{\mathrm{RMS}}$ to make sure stable power frequency $\mathrm{AC}$ supply.

Table I summarizes the information about the different components of the loop.

TABLE I

COMPONENTS OF THE TEST LOOP

\begin{tabular}{|c|c|c|c|}
\hline Components & Material & Quantity & Size \\
\hline $\begin{array}{l}\text { Bolted power } \\
\text { connector }\end{array}$ & A356.0 alloy & 1 & $\begin{array}{c}\text { Length }=0.075 \mathrm{~m} \\
\text { Inner Diameter }=0.040 \mathrm{~m}\end{array}$ \\
\hline Cylindrical bus bar & Aluminum & 2 & $\begin{array}{c}\text { Length }=1 \mathrm{~m} \\
\text { Diameter }=0.040 \mathrm{~m}\end{array}$ \\
\hline Bolts and nuts & Steel & $\begin{array}{l}8 \\
6\end{array}$ & $\begin{array}{l}\text { M7 x } 20 \mathrm{~mm} \\
\mathrm{M} 10 \times 50 \mathrm{~mm}\end{array}$ \\
\hline $\begin{array}{l}\text { Flexible cables with } \\
\text { terminals }\end{array}$ & Aluminum & 3 & $\begin{array}{c}\text { Length }=1 \mathrm{~m} \\
\text { Diameter }=0.02 \mathrm{~m}\end{array}$ \\
\hline
\end{tabular}

According to the flowcharts summarized in Figs. 3 and 4 , the measurement of the contact resistance under both DC and $\mathrm{AC}$ power frequency supply, requires a previous measurement of the initial contact resistance. The reference DC resistance $R_{D C}$ of the power connector was measured by means of the 4wire method, as detailed in Section II. A digital microohmmeter (Raytech Micro-Centurion II, 0-200 $\mathrm{A}_{\mathrm{DC}}, \pm 0.01 \mu \Omega$ ) was used to this end.

From this measurement, the initial DC resistance of the connector resulted in $R_{\text {Connector, } 20^{\circ} \mathrm{C}}=111.8 \mu \Omega$. Next, from FEM simulations the bulk resistance was found to be $R_{b u l k}=$ $4.86 \pm 0.01 \mu \Omega$. Hence, $\Delta u_{R_{B u l k}}=0.21 \%$. Finally, by applying (2) the resulting initial contact resistance was $R_{\text {Contact }, D C}=R_{\text {Connector }, 20^{\circ} \mathrm{C}}-R_{\text {Bulk }}=106.9 \mu \Omega$, which was taken as the reference value of the contact resistance. In per-unit system, as usually applied in power system analysis, it becomes $R_{\text {Contact,DC, } p . u \text {. }}=1$.

\section{A. Contact Resistance Measurement under DC Supply}

This section evaluates the accuracy of the method proposed in Fig. 3 to measure the contact resistance of the power connector under DC supply. It is noted that the accuracy of the DC measurement can be affected by the thermoelectric electromotive force between the metallic wires used to measure the voltage drop across the terminals of the connector. Since the connector is made of aluminum, the thermoelectric effect was minimized by using aluminum wires, although a residual electromotive force is generated between those wires and the terminals of the DAQ, where the wires are connected. Since for most paired materials this voltage is in the order of some $\mu \mathrm{V} /{ }^{\circ} \mathrm{C}$, to minimize its effect it is necessary a voltage drop $I \cdot R_{\text {Connector }}$ of at least some $\mathrm{mV}$.

The experimental loop shown in Fig. 5 was tested by applying different DC current levels, and the contact resistance was measured according to the procedure detailed in Section III.A and Fig. 3.

Fig. 6 depicts the voltage drop across the connector under DC supply for the different levels of current applied in the experimental tests. The slope of this curve provides information about the total DC resistance of the connector, which includes the bulk and the contact resistances. It shows a very linear behavior, thus allowing to determine the contact resistance.

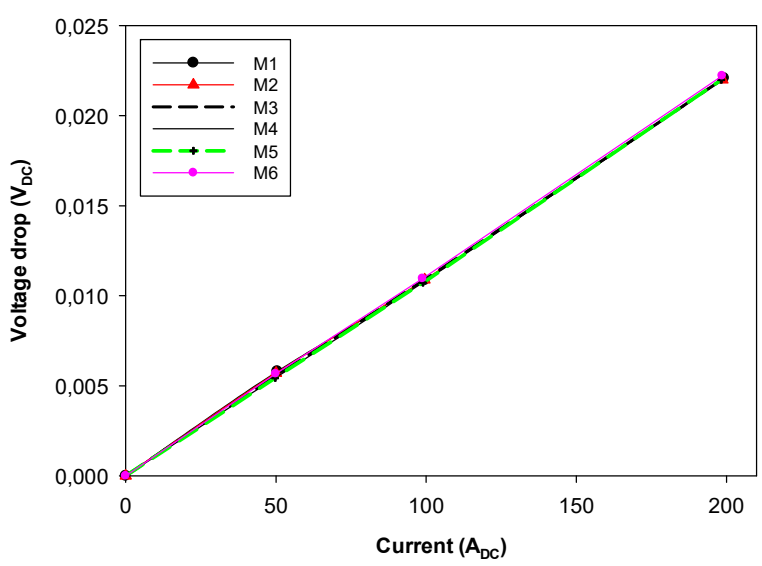

Fig. 6. Voltage drop across the terminals of the connector under DC supply for different current levels. M1 to M6 correspond to six sets of measurements, each one including four current levels $\left(0,50,100\right.$ and $\left.200 \mathrm{~A}_{\mathrm{DC}}\right)$.

Table II shows the contact resistance measurements performed under DC supply when applying different current levels.

TABLE II

EXPerimental Values Of The Contact Resistance In Per Unit Under DC SUPPLY AND ERROR EVALUATION

\begin{tabular}{|c|c|c|c|c|c|c|c|c|}
\hline $\begin{array}{c}\text { Current } \\
\left(\mathrm{A}_{\mathrm{DC}}\right)\end{array}$ & Cor & act res & tonco & .u. $)=1$ & rror (p. & & $\begin{array}{c}\text { Mean } \\
R_{\text {Contact.p.u. }}\end{array}$ & $\begin{array}{l}\text { Mean } \\
\text { error }\end{array}$ \\
\hline & M1 & M2 & M3 & M4 & M5 & M6 & & \\
\hline 50 & 1.068 & 1.060 & 1.026 & 1.009 & 1.007 & 1.049 & 1.037 & $3.7 \%$ \\
\hline 100 & 1.012 & 1.015 & 1.013 & 1.002 & 1.000 & 1.023 & 1.011 & $1.1 \%$ \\
\hline 200 & 1.028 & 1.027 & 1.025 & 1.023 & 1.024 & 1.033 & 1.027 & $2.7 \%$ \\
\hline
\end{tabular}

M1.M2.M3.M4.M5.M6: set of DC measurements done

Results summarized in Table II indicate that the experimental mean error in measuring the contact resistance under DC supply is below 4\%. It is concluded that such results are accurate enough for an online estimation of the contact resistance in order to assess the health condition of the connector.

\section{B. Contact Resistance Measurement under AC Supply}

This section assesses the accuracy of the method proposed in Fig. 4 for an experimental measurement of the contact resistance under power frequency AC supply.

The initial DC contact resistance of the connector was measured during the calibration stage, following the procedure 
detailed in Section II. It is a recognized fact that the AC resistance can be significantly different from the DC resistance due to the effect of eddy currents, which produce an uneven distribution of the current density across the crosssection of the connector. In the analyzed case, the skin effect predominates over the proximity effect, although the latter one can be important under the presence of nearby conductors and connectors [37]. This paper proposes determining the $k=$ $R_{\text {Connector.AC }} / R_{\text {Connector.DC }}$ ratio by applying FEM simulations. since FEM models can be applied to a wide range of complex configurations and electrical frequencies [7], [37]. FEM results show that for the analyzed configuration, the former ratio results in $R_{\text {Contact.AC.p.u. }}=1.127 \cdot R_{\text {Contact.DC.p.u. }}$ The contact resistance of the connector under power frequency AC supply was determined from experimental tests in the loop depicted in Fig. 5. For this purpose, different current levels were applied in the range of $0.5-1.0 \mathrm{kA}_{\mathrm{RMS}}$. The contact resistance was measured online by applying the approach summarized in Fig. 4.

Fig. 7 depicts the voltage drop across the connector under power frequency AC supply for the different levels of current applied in the experimental tests. It shows a very linear behavior, thus allowing a precise determination of the contact resistance.

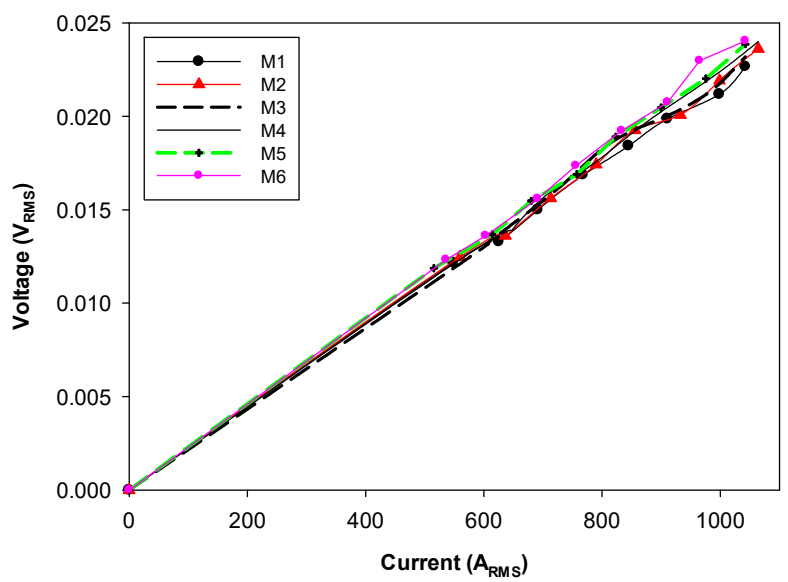

Fig. 7. Voltage drop across the terminals of the connector under power frequency AC supply for different current levels. M1 to M6 correspond to six sets of measurements, including nine current levels, each one within the range $0-1050$ A RMS.

Table III summarizes the contact resistance measurements performed under power frequency AC supply when applying different levels of current.

Results shown in Table III indicate that the experimental mean error in the contact resistance measurement under power frequency AC supply is below $2.5 \%$.

It is concluded that such results are accurate enough for an online estimation of the contact resistance to determine the health condition of the connector, as this paper proposes a limit of acceptable resistance increase of at least $20 \%$, which is in agreement with the IEC 62271-1:2017 standard [38].

$$
\text { TABLE III }
$$

EXPERIMENTAL VALUeS OF THE CONTACT RESISTANCE UNDER POWER FREQUENCY AC SUPPLY AND ERROR EVALUATION

\begin{tabular}{lccc}
\hline \hline Current & Contact resistance (p.u.) & Mean & Mean
\end{tabular}

\begin{tabular}{|c|c|c|c|c|c|c|c|c|}
\hline$\left(\mathrm{kA}_{\mathrm{RMS}}\right)$ & & & & & & & $R_{\text {Contact.p.u. }}$ & error \\
\hline & M1 & M2 & M3 & M4 & M5 & M6 & & \\
\hline$\approx 0.54$ & 1.063 & 1.046 & 0.960 & 1.011 & 1.006 & 1.023 & 1.018 & $1.8 \%$ \\
\hline$\approx 0.62$ & 1.000 & 0.954 & 0.971 & 0.971 & 0.960 & 0.994 & 0.975 & $2.5 \%$ \\
\hline$\approx 0.68$ & 1.017 & 1.023 & 0.983 & 1.029 & 0.994 & 0.989 & 1.006 & $0.6 \%$ \\
\hline$\approx 0.74$ & 1.023 & 1.011 & 1.017 & 1.011 & 0.971 & 1.017 & 1.009 & $0.9 \%$ \\
\hline$\approx 0.82$ & 1.023 & 1.040 & 1.023 & 1.023 & 1.006 & 1.017 & 1.022 & $2.2 \%$ \\
\hline$\approx 0.88$ & 1.011 & 0.960 & 0.966 & 1.011 & 0.994 & 1.000 & 0.990 & $1.0 \%$ \\
\hline$\approx 0.96$ & 0.966 & 0.966 & 0.960 & 1.000 & 0.989 & 1.052 & 0.989 & $1.1 \%$ \\
\hline$\approx 1.02$ & 0.989 & 0.994 & 0.977 & 1.006 & 1.011 & 0.994 & 0.995 & $0.5 \%$ \\
\hline
\end{tabular}

\section{CONTACt Resistance UnCERTAINTy AnAlysis}

\section{A. DC supply}

As shown in (10), the contact resistance under DC supply has different components, that is, $R_{\text {Bulk }}$ obtained from FEM simulations, $\Delta V_{\text {Connector }}$ measured by means of voltage measuring device and $I_{\text {Connector }}$ measured by means of the Hall sensor.

$$
R_{\text {Contact }}=R_{\text {Connector }}-R_{\text {Bulk }}=\frac{\Delta V_{\text {Connector }}}{I_{\text {Connector }}}-R_{\text {Bulk }}
$$

The uncertainty of $R_{\text {Contact }}$, here expressed as $\Delta u_{R_{\text {Contact }}}$, can be calculated as,

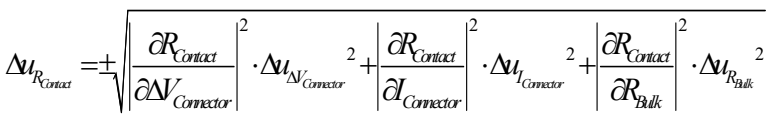

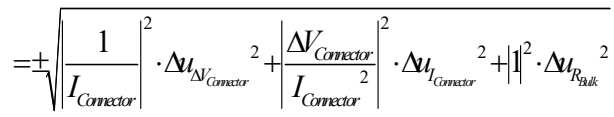

The uncertainty of the voltage measuring device is $1.35 \%$ according to [39] but this value is corrected to $3.2 \%$, since the error is more at low input voltage. So, $\Delta u_{\Delta V_{\text {Connector }}}$ is $3.2 \%$.

The total uncertainty of the current has several components like alignment, thermoelectric voltage, voltage offset, or environmental conditions among others, thus depending on installation conditions. Therefore, an in-situ calibration method is suggested in this paper. The accuracy of the current measurement provided by the Hall sensor was calculated by comparing the current measured by this sensor with that generated by a calibrated DC current source. Since,

$$
I_{\text {Connector }}=I_{\text {CurrentSource }}+\Delta I_{D C}
$$

the uncertainty of $I_{\text {Connector }}$ can be calculated as,

$$
\Delta u_{I_{\text {Comeretor }}}= \pm \sqrt{\Delta u_{I_{\text {Curreussoure }}}{ }^{2}+\Delta u_{\Delta I_{D C}}{ }^{2}}
$$

including uncertainty of the calibrated DC source and uncertainty of Hall sensor with respect to the calibrated DC source.

\section{B. AC supply}

The proposed system for AC analysis is made up of four different parts. i.e. $R_{\text {Bulk }}$ from FEM simulations, $\Delta V_{\text {Connector }}$ from voltage measuring device, $I_{\text {Connector }}$ from Hall sensor and $\cos \varphi$ which is the phase difference between $\Delta V_{\text {Connector }}$ and $I_{\text {Connector }}$. Hence, the combined standard uncertainty of $R_{\text {Contact }}$ can be calculated as shown below in equation 14 . 


$$
\begin{aligned}
& R_{\text {catat }}=R_{\text {conetar }}-R_{\text {Bdlk }}=\frac{\Delta V_{\text {Gametar }}}{I_{\text {Comedar }}} \cdot \cos \varphi-R_{\text {adk }}
\end{aligned}
$$

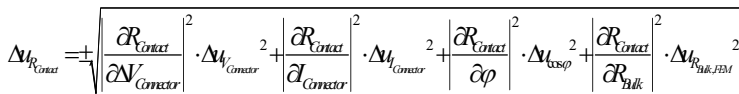

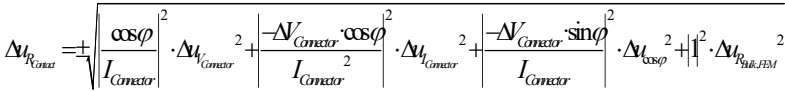

The accuracy of the current measurement provided by the Hall sensor was calculated by comparing the current measured by this sensor with that generated by a calibrated Rogowski coil.

$$
I_{\text {Connector }}=I_{\text {Rogowski }}+\Delta I_{A C}
$$

the uncertainty of $I_{\text {Connector }}$ can be calculated as,

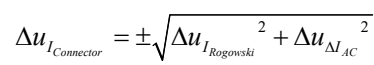

including uncertainty of the calibrated Rogowski coil and uncertainty of Hall sensor with respect to the calibrated Rogowski coil.

\section{Uncertainty results}

The maximum uncertainties of the individual components and the entire system is calculated by substituting the values of experimental data in the equations (10) - (16). Results are summarized in below Table VII.

TABLE VII

SUMMARY OF THE UNCERTAINTIES INTRODUCED IN THE SYSTEM

\begin{tabular}{ccc}
\hline \hline Components & \multicolumn{2}{c}{ Accuracy $(\%)$} \\
\hline DC & AC \\
\hline $\begin{array}{c}\text { Calibrated DC source }\left(\Delta u_{I_{\text {CurrentSource }}}\right) \\
\text { Hall Sensor with respect to DC source }\end{array}$ & $0.10^{\mathrm{a}}$ & 0.33 \\
and Rogowski coil $\left(\Delta u_{\Delta I}\right)$ & 1.19 & 1.90 \\
Phase $\left(\Delta u_{\cos \varphi}\right)$ & \\
Hall Sensor $\left(\Delta u_{I_{\text {Connector }}}\right)$ & - & 2.77 \\
National Instrument $\left(\Delta u_{\Delta V_{\text {Connector }}}\right)$ & 1.19 & 1.92 \\
FEM Simulation $\left(\Delta u_{R_{\text {Bulk }, \text { FEM }}}\right)$ & 0.21 & 3.20 \\
Entire System $\left(\Delta u_{R_{\text {Contact }}}\right)$ & 3.76 & 0.21 \\
\hline \hline
\end{tabular}

${ }^{\text {a }}$ From the datasheet of the Raytech Micro Centurion II [40]

\section{CONCLUSION}

Measurements done by means of distributed sensors and wireless communication systems eases predictive maintenance tasks and online assessment of the condition of in-service equipment. This approach is a trending topic in predictive maintenance of power systems, as it allows reducing unexpected failures and unplanned disconnections, which can lead to severe consequences. Currently, health condition models of power connectors are not available, although it is believed that they can be a cost-effective tool to increase power systems reliability. The contact resistance is a reliable indicator of the health condition of high-voltage power connectors, so this paper has proposed a low-cost system for online measurement of contact resistance. Contact resistance cannot be measured directly, so a hybrid experimental and simulation based approach has been proposed, which is applicable for both DC and AC power systems. Since the goal is determining the medium-term evolution of the contact resistance, the accuracy of this measurement is not a critical issue. Experimental results show the accuracy and feasibility of the proposed approach for both $\mathrm{DC}$ and $\mathrm{AC}$ cases, the accuracy being better than $3-5 \%$. It is noted that the proposed approach can also be applied to many other power systems' elements integrating electrical contacts.

\section{ACKNOWLEDGMENT}

This work was partially supported by the Generalitat de Catalunya under the project 2017 SGR 967 and 2016 DI 065, respectively.

\section{REFERENCES}

[1] F. Capelli, J.-R. J.-R. Riba, and J. Sanllehí, "Finite element analysis to predict temperature rise tests in high-capacity substation connectors," IET Gener. Transm. Distrib., vol. 11, no. 9, pp. 2283-2291, Jun. 2017.

[2] F. de Paulis, C. Olivieri, A. Orlandi, and G. Giannuzzi, "Detectability of Degraded Joint Discontinuities in HV Power Lines Through TDR-Like Remote Monitoring," IEEE Trans. Instrum. Meas., vol. 65, no. 12, pp. 2725-2733, Dec. 2016.

[3] V. Pascucci et al., "A Standardized Reliability Evaluation Framework for Connections," in SMTA International, 2016, pp. 1-10.

[4] E. Carvou, R. El Abdi, J. Razafiarivelo, N. Benjemaa, and E. M. Zindine, "Thermo-mechanical study of a power connector," Measurement, vol. 45, no. 5, pp. 889-896, Jun. 2012.

[5] R. Tzeneva, "Investigation of High Power Bolted Busbar Connectors with Longitudinal Slots," Przeglad Elektrotechniczny, pp. 223-226, 2012.

[6] W. E. Wilson, S. V. Angadi, and R. L. Jackson, "Surface separation and contact resistance considering sinusoidal elastic-plastic multi-scale rough surface contact," Wear, vol. 268, no. 1-2, pp. 190-201, Jan. 2010.

[7] J.-R. Riba, A.-G. Mancini, C. Abomailek, and F. Capelli, "3D-FEMBased Model to Predict the Electrical Constriction Resistance of Compressed Contacts," Measurement, vol. 114, no. January 2018, pp. 44-50, 2018.

[8] I. M. G. González-Díaz, D. Pastor, E. García-Hemme, D. Montero, R. García-Hernansanz, J. Olea, A. del Prado, E. San Andrés, "A robust method to determine the contact resistance using the van der Pauw set up," Measurement, vol. Online, pp. 1-18, 2017.

[9] S. Kasi and L. Herron, "Theory of the Effect of Torque and Re-Torque Practices on Electrical Connectors with Clamping Fasteners," in Power India International Conference (PIICON), 2016 IEEE 7th, 2016, pp. 16.

[10] P. G. Slade, Electrical Contacts: Principles and Applications, Second Edition. 2017.

[11] International Electrotechnical Commission, "IEC TS 61586:2017 Estimation of the reliability of electrical connectors.” IEC, pp. 1-55, 2017.

[12] F. Capelli, J. Riba, E. Ruperez, and J. Sanllehi, "A Genetic-AlgorithmOptimized Fractal Model to Predict the Constriction Resistance From Surface Roughness Measurements," IEEE Trans. Instrum. Meas., vol. 66, no. 9, pp. 2437-2447, 2017.

[13] C. Zhai, D. Hanaor, G. Proust, and Y. Gan, "Stress-Dependent Electrical Contact Resistance at Fractal Rough Surfaces," J. Eng. Mech., no. 5, p. B4015001, 2015.

[14] M. Ciavarella, G. Murolo, and G. Demelio, "The electrical/thermal conductance of rough surfaces - the Weierstrass-Archard multiscale model," Int. J. Solids Struct., vol. 41, pp. 4107-4120, 2004.

[15] D. Gonzalez, F. Berger, M. Hopfeld, and P. Schaaf, "Model switch experiments for determining the evolution of contact resistance of electrical contacts in contactors," in 2016 IEEE 62nd Holm Conference on Electrical Contacts (Holm), 2016, pp. 129-134.

[16] F. Capelli, J.-R. Riba, and D. Gonzalez, "Thermal behavior of energyefficient substation connectors," in 10th International Conference on Compatibility, Power Electronics and Power Engineering (CPEPOWERENG), 2016, pp. 104-109.

[17] D. Gonzalez, M. Hopfeld, F. Berger, and P. Schaaf, "Investigation on Contact Resistance Behavior of Switching Contacts Using a Newly Developed Model Switch," IEEE Trans. Components, Packag. Manuf. 
Technol., vol. 8, no. 6, pp. 939-949, Jun. 2018.

[18] Q. Shen, K. Lv, G. Liu, and J. Qiu, "Dynamic Performance of Electrical Connector Contact Resistance and Intermittent Fault Under Vibration,' IEEE Trans. Components, Packag. Manuf. Technol., vol. 8, no. 2, pp. 216-225, Feb. 2018.

[19] Q. Shen, K. Lv, G. Liu, and J. Qiu, "Impact of Electrical Contact Resistance on the High-Speed Transmission and On-Line Diagnosis of Electrical Connector Intermittent Faults," IEEE Access, vol. 5, pp. 4221-4232, 2017.

[20] C. Farges, M. Chevrie, J. Sabatier, L. Pradere, and F. Guillemard, "Detection of Electric Contact Resistance Variations in Automotive Connectors," IEEE Trans. Ind. Electron., vol. 64, no. 12, pp. 9469 9476, Dec. 2017.

[21] J. Qu, X. Li, and Q. Wang, "Experimental study on electric resistance of tilted contact in air circuit breaker," in 2016 IEEE 62nd Holm Conference on Electrical Contacts (Holm), 2016, pp. 115-118.

[22] Z. Li, Q. Li, Z. Wu, J. Yu, and R. Zheng, "A Fault Diagnosis Method for On Load Tap Changer of Aerospace Power Grid Based on the Current Detection," IEEE Access, vol. 6, pp. 24148-24156, 2018.

[23] J. S. Bobowski, M. S. Ferdous, and T. Johnson, "Calibrated SingleContact Voltage Sensor for High-Voltage Monitoring Applications," IEEE Trans. Instrum. Meas., vol. 64, no. 4, pp. 923-934, Apr. 2015.

[24] A. Grima, A. Danisi, A. Masi, and N. Sammut, "Influence of External Conductive Objects on the Performance of an Ironless Inductive Position Sensor," IEEE Sens. J., vol. 17, no. 14, pp. 4500-4507, Jul. 2017.

[25] Y. Hu, Y. Yan, L. Wang, and X. Qian, "Non-Contact Vibration Monitoring of Power Transmission Belts Through Electrostatic Sensing," IEEE Sens. J., vol. 16, no. 10, pp. 3541-3550, May 2016.

[26] C. Zachariades, R. Shuttleworth, R. Giussani, and R. MacKinlay, "Optimization of a High-Frequency Current Transformer Sensor for Partial Discharge Detection Using Finite-Element Analysis," IEEE Sens. J., vol. 16, no. 20, pp. 7526-7533, Oct. 2016.

[27] A. Laor, P. J. Herrell, and M. Mayer, "A Study on Measuring Contact Resistance of Ball Bonds on Thin Metallization," IEEE Trans. Components, Packag. Manuf. Technol., vol. 5, no. 5, pp. 704-708, May 2015 .

[28] E. Grundkotter, P. Weskamp, and J. Melbert, "Transient ThermoVoltages on High-Power Shunt Resistors," IEEE Trans. Instrum. Meas., vol. 67, no. 2, pp. 415-424, Feb. 2018.

[29] T. Asada, W. G. Odendaal, and J. D. van Wyk, "An overview of integratable current sensor technologies," 38th IAS Annu. Meet. Conf. Rec. Ind. Appl. Conf. 2003., vol. 2, pp. 1251-1258, 2003.

[30] F. Xie, R. Weiss, and R. Weigel, "Giant-Magnetoresistance-Based Galvanically Isolated Voltage and Current Measurements," IEEE Trans. Instrum. Meas., vol. 64, no. 8, pp. 2048-2054, 2015.

[31] S. D. T. Dewi, C. Panatarani, and I. M. Joni, "Design and development of DC high current sensor using Hall-Effect method," AIP Conf. Proc., vol. 1712, pp. 1-6, 2016.

[32] R. Portas and L. Colombel, "Accuracy of Hall-Effect Current Measurement Transducers in Automotive Battery Management Applications using Current Integration," pp. 1-8, 2007.

[33] F. Capelli, J.-R. Riba, and J. Pérez, "Three-Dimensional Finite-Element Analysis of the Short-Time and Peak Withstand Current Tests in Substation Connectors," Energies, vol. 9, no. 6, p. 418, May 2016.

[34] K. Wang, G. Gong, G. Chen, Y. Xiao, and H. Lu, "Online Measurement Method of Phase Difference of Current Sensor based on CCA," 2017, no. Icsai, pp. 242-246.

[35] E. Kona, "Stationary VRLA battery health estimation by resistance measurement - comparison of dc and ac test methods," 2016 IEEE Int. Conf. Power Electron. Drives Energy Syst., pp. 1-5, 2016.

[36] D. Belega, D. Petri, and D. Dallet, "Amplitude and Phase Estimation of Real-Valued Sine Wave via Frequency-Domain Linear Least-Squares Algorithms," pp. 1-13, 2018.

[37] J.-R. Riba, "Analysis of formulas to calculate the AC resistance of different conductors' configurations," Electr. Power Syst. Res., vol. 127, 2015 .

[38] International Electrotechnical Commission, "IEC 62271-1:2017. Highvoltage switchgear and controlgear - Part 1: Common specifications for alternating current switchgear and controlgear." International Electrotechnical Commission, p. 260, 2017.

[39] National Instruments, "Specifications USB-6000 8 AI (10 kS/s), 4 DIO USB Multifunction I/O Device.” pp. 1-8, 2018.

[40] Raytech, "Micro-Centurion-II." [Online]. Available: http://www.raytech.ch/Products/Micro-Ohm/Micro-Centurion-II. 